\title{
Hidrops fetal por infección congénita por citomegalovirus: tratamiento posneonatal prolongado con valganciclovir
}

\author{
Silvia Campos Arca ${ }^{1}$, María Fernández Rial ${ }^{1}$, Isabel Pardo Pumar ${ }^{1}$, Cristina \\ Luaces $^{2}$, José García Piñó ${ }^{2}$, Eloy Moral Santamarina ${ }^{1}$. \\ 1 Servicio de Ginecología y Obstetricia, ${ }^{2}$ Servicio de Pediatría, Complejo Hospitalario de Pontevedra. Pontevedra. \\ España.
}

\section{RESUMEN}

El citomegalovirus (CMV) es la infección viral congénita más frecuente con una prevalencia de 0,5\% al nacimiento. La primoinfección aparece entre el 1-4\% de las gestantes seronegativas. El $40 \%$ de estos fetos se infectan y un $10 \%$ presentan síntomas al nacimiento. Presentamos un caso de infección congénita por CMV con hidrops fetal, con afectación neonatal del sistema nervioso central. Se trató con ganciclovir intravenoso y posteriormente con valganciclovir oral hasta los 6 meses, con buenos resultados al año de vida. Se realiza una revisión bibliográfica del diagnóstico y pronóstico de los recién nacidos con infección congénita por CMV y las expectativas y experiencia actual del tratamiento con ganciclovir y valganciclovir.

PALABRAS CLAVE: Infección congénita, citomegalovirus congénito, ganciclovir, valganciclovir

\section{SUMMARY}

Cytomegalovirus (CMV) is the leading cause of congenital infection affecting $0.5 \%$ of all live births. Primary CMV infection occurs in 1-4\% of seronegative woman during pregnancy and may be transmitted to the fetus in $40 \%$. Up to $10 \%$ of intrauterine CMV infections result in symptomatic congenital disease at birth. We present a case of congenital CMV infection in the third trimester of gestation with central nervous disease involvement, who was treated with intravenosus ganciclovir followed by oral valganciclovir for six months with successful results in the first year of life. We review the literature on the diagnosis and prognosis of newborns with congenital CMV infection and the expectations and current experience of treatment with ganciclovir and valganciclovir.

KEY WORDS: Congenital infection, congenital cytomegalovirus, ganciclovir, valganciclovir

\section{INTRODUCCIÓN}

El Citomegalovirus (CMV) produce la infección congénita más frecuente en nuestro medio. Oscila entre el $0,2-2,4 \%$ de los recién nacidos (1-6), y es una causa importante de secuelas neurológicas y sensoriales (7).

Se presenta un caso de infección congénita por CMV con hidrops fetal y recién nacido sintomático que fue tratado con ganciclovir y valganciclovir de durante 6 meses, con buenos resultados al año de vida. 


\section{Caso clínico}

Gestante de 19 años, de etnia gitana, primigesta, sin antecedentes de importancia. Evolución fisiológica hasta la semana 26. Posteriormente sin control prenatal. En la semana $39+3$ acude de urgencia por sensación de dinámica uterina dolorosa. En la ecografía al ingreso se observa presencia de un feto con hidrops importante a expensas de ascitis y derrame pericárdico con cardiomegalia (Figura 1).

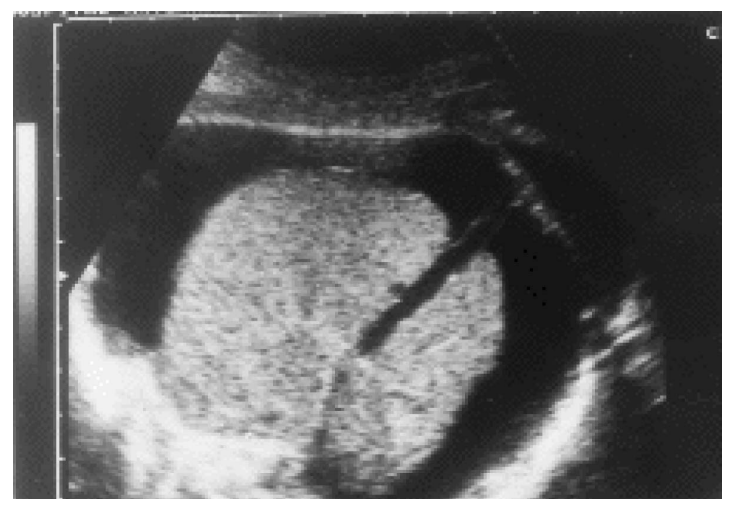

Figura 1. Imagen ecográfica de ascitis fetal.

Se decide traslado a centro de tercer nivel, donde realizan una cesárea de urgencia. Nace un recién nacido varón con edema y ascitis, escaso esfuerzo respiratorio, hipotonía, bradicardia y cianosis, que precisa maniobras de reanimación cardiopulmonar neonatal avanzada. Apgar 6-8 (intubado). Se conecta a ventilación mecánica en periodo neonatal inmediato. Peso al nacer de 3.156 gramos (percentil 25), perímetro craneal $29 \mathrm{~cm}(<2$ desviaciones estándar). Presenta tinte ictérico de piel y mucosas, petequias y lesiones purpúricas en cara, tórax, abdomen y extremidades, sin evidencia de malformaciones externas mayores. Hepatomegalia de $5 \mathrm{~cm}$ y esplenomegalia de $6 \mathrm{~cm}$, con signo de la oleada positivo. Hidrocele bilateral.

Ingresa en $\mathrm{UCl}$ neonatal donde se realizan estudios complementarios. Analítica: Leucocitos $51.090 / \mathrm{mm}^{3}$ con linfocitos de $77 \%$; hemoglobina $15,4 \mathrm{~g} / \mathrm{dl}$; hematocrito $47,3 \%$; plaquetas $14.000 /$ $\mathrm{mm}^{3}$; bilirrubina total a las 48 horas de $15,3 \mathrm{mg} / \mathrm{dl} \mathrm{y}$ trasaminasas altas (GOT $404 \mathrm{UI} / \mathrm{L}$ y GPT $97 \mathrm{UI} / \mathrm{L}$ ). Serología: IgM CMV negativo. Cultivo Shell-vial positivo en orina. PCR ADN CMV positivo en sangre (18.100 copias/ml) y negativo en líquido cefalorraquídeo.

Imágenes: La ecografía trasfontanelar muestra calcificaciones parenquimatosas, vasculopatía dentículo- estriada bilateral y discreta dilatación del sistema ventricular. La ecografía abdominal muestra hepatoesplenomegalia homogénea con calcificación hepática puntiforme y ascitis. El ecocardiograma muestra hipertensión pulmonar con ventrículo derecho dilatado y derrame pericárdico leve moderado. La resonancia magnética cerebral muestra leve dilatación de las astas de los ventrículos laterales y focos de hiperseñal en la sustancia blanca que podrían estar en relación con leucomalacia (Figura 2).

Otros: Electrocardiograma y electroencefalograma sin alteraciones. Potenciales evocados auditivos y visuales normales. Exploración oftalmológica y auditiva normal.

Serología materna al parto: CMV IgG positiva e IgM negativa.

El estudio anatomopatológico de la placenta muestra vellosidades con grupos de células plasmáticas intravellositarias que sugiere infección por $\mathrm{CMV}$, no se observan inclusiones virales.

Se efectúa el diagnóstico de infección congénita por CMV, shock séptico, hipertensión pulmonar secundaria, ascitis moderada, derrame pericárdico leve y microcefalia congénita.

Se administra gammaglobulina humana inespecífica por tres días y gammaglobulina anti-CMV tres dosis. Se inicia tratamiento con ganciclovir intravenoso (12 mg/kg/día) que se completa por 6 semanas, con leucopenia y neutropenia secundarias, tras lo cual se inicia tratamiento con valganciclovir oral de $32 \mathrm{mg} / \mathrm{kg} /$ día en dos dosis durante 6 meses. Desde el inicio del tratamiento se objetiva disminución de la carga viral en sangre y orina hasta hacerse indetectable, con normalización de las transaminasas.

A los 2 meses de vida se decide alta hospitalaria con el diagnóstico de infección congénita por CMV y alteración del desarrollo psicomotor moderado. Se inicia seguimiento multidisciplinario por pediatría, neurología, oftalmología y otorrinolaringología, con rehabilitación precoz desde el alta.

Actualmente el niño tiene 1 año y dos meses de vida. La evolución neurológica ha sido favorable, siendo normal la exploración neurológica al año de vida, demostrándose por el momento sólo hipoacusia unilateral moderada desde los ocho meses de edad.

\section{DISCUSIÓN}

Las infecciones son responsables del $4 \%$ de los hidrops fetales (8). La infección por CMV es una de las causas más frecuentes de retraso psicomotor y sordera neurosensorial de origen infeccioso. En España se estima una tasa de 0,5\% (9), aunque 


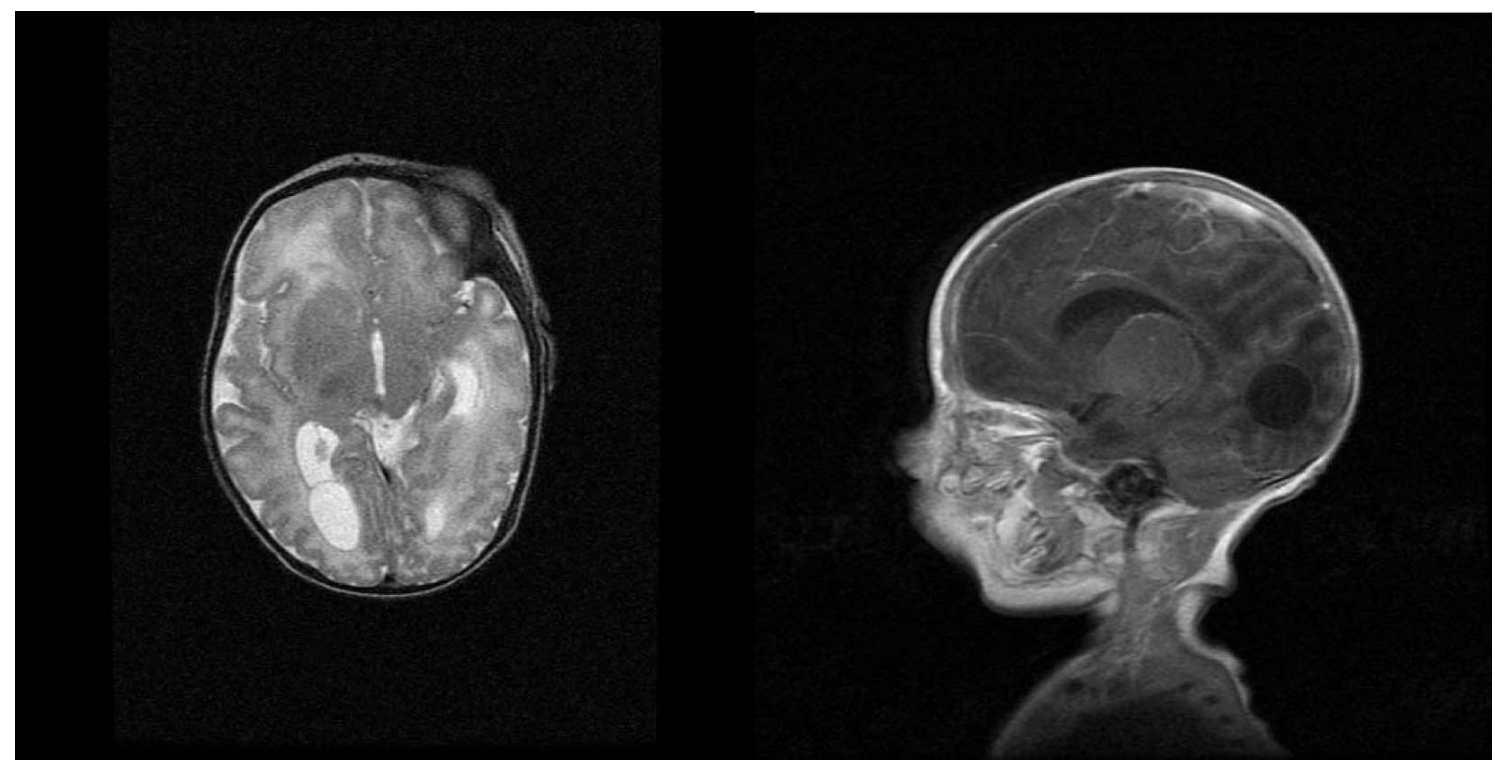

Figura 2. Imágenes de RMN cerebral neonatal. Cortes trasversal y sagital donde se observa adelgazamiento del cuerpo calloso y dilatación de los ventrículos laterales, hemorragia intraventricular en asta posterior del ventrículo izquierdo. Focos de hiperseñal en la sustancia blanca periventricular en relación con probable leucomalacia.

desconocemos la prevalencia de la infección congénita en el recién nacido $(10,11)$.

La Sociedad Española de Ginecología y Obstetricia no recomienda la realización de cribado serológico sistemático frente a CMV durante el embarazo (12-15). Las pruebas serológicas para el CMV pueden ser consideradas para madres con primoinfección demostrada en el embarazo o cuadro pseudomononucleósido con serología compatible; a los niños con infección fetal demostrada o alteraciones ecográficas indicativas; la presencia al nacimiento de clínica compatible. También se recomienda a trabajadoras de educación infantil o mujeres con niño en la guardería y madres VIH positivas; en recién nacidos prematuros, especialmente menores a las 32 semanas o peso inferior a 1500 gramos; neonatos con crecimiento intrauterino retardado (15).

Las mujeres embarazadas que son susceptibles a la infección por CMV deben ser advertidas de la importancia de lavarse las manos y la limpieza de las superficies ambientales al estar con niños pequeños. Igualmente, si trabajan en una guardería o ambiente preescolar, deben utilizar guantes para cambiar los pañales de los niños, así como lavarse las manos después de cada cambio de pañal y de manipular los juguetes u otros objetos que los niños podrían haber colocado en la boca (15).
La primoinfección en la embarazada suele ser asintomática, aunque un $30 \%$ cursa con fiebre prolongada, cuadro seudogripal o mononucleósido (16). La gravedad de la infección es mayor en el primer y segundo trimestre, mientras que la posibilidad de transmisión es mayor en el tercer trimestre $(18,19)$. La presencia de IgG positiva en ausencia de IgM es el hallazgo más frecuente (10), ya que IgM suele negativizarse en 3-4 meses (20). Lo más útil es la avidez de la lgG, una baja avidez indicaría infección reciente en los 3-6 meses previos (21-22).

El mejor método diagnóstico de infección fetal es la PCR de líquido amniótico, que presenta una sensibilidad de $90-98 \%(15,23)$ y especificidad de $92-98 \%(20-21)$. Los exámenes ecográficos seriados, se deben realizar cada 2-4 semanas, aunque es importante tener en cuenta que la ausencia de hallazgos ecográficos no garantiza un resultado normal (15). Los hallazgos ecográficos de infección congénita son la presencia de hidrops fetal y las calcificaciones cerebrales, el retraso de crecimiento, la hepatoesplenomegalia, el aumento de la ecogenidad intestinal, la microcefalia, la dilatación ventricular y la atrofia cortical (24-29).

El método diagnóstico más utilizado en el recién nacido es el cultivo de virus en orina shell viral por su rapidez y su alta especificidad (21). El estudio 
anatomopatológico de la placenta puede contribuir al diagnóstico.

Al nacimiento se realiza exploración neurológica completa, analítica con función hepática, punción lumbar, ecografía cerebral, fondo de ojo, potenciales evocados auditivos y visuales. Se recomienda completar estudios con resonancia magnética nuclear cerebral por tener mayor sensibilidad para el diagnóstico de displasias corticales y lesiones en la sustancia blanca (7).

Uno de los aspectos que más nos interesa en estos recién nacidos, es el pronóstico a largo plazo y la posibilidad de predecir que niños van a tener más posibilidades de secuelas. Estas son más frecuentes en recién nacidos sintomáticos, como en este caso (3). Las principales secuelas son la sordera neurosensorial y la hipoacusia, que es bilateral en dos tercios de los niños y puede ser progresiva (30-33). Otras secuelas son el retraso psicomotor, parálisis cerebral, hipotonía, paresia, epilepsia, ceguera cortical o coriorretinitis, retraso del aprendizaje y lenguaje $(7,18)$. El riesgo de retraso psicomotor es más frecuente cuando hay alteraciones en la neuroimagen detectadas en el primer mes de vida $(33,34)$. Sin embargo, el desarrollo de hipoacusia neurosensorial ocurre prácticamente igual en niños sintomáticos con o sin afectación nerológica (33). La evolución neurológica de estos niños con infección sintomática es muy variable y poco predecible al nacimiento (35).

La Asociación Española de Pediatría recomienda tratar con ganciclovir a todos los neonatos con infección congénita y afectación del sistema nervioso central para prevenir el desarrollo de hipoacusia, alteraciones del lenguaje y aprendizaje, y mejora también la trombocitopenia, la hepatitis colestásica y la coriorretinitis $(7,36)$. Durante el embarazo el tratamiento antiretroviral no se recomienda (3739), pero sí la administración de gammaglobulina humana anti-CMV en mujeres con primoinfección demostrada $(24,40)$.

En el recién nacido con infección congénita sintomática por CMV, el ganciclovir intravenoso (12 $\mathrm{mg} / \mathrm{kg} / \mathrm{día}$ ) en dos dosis durante 6 semanas y el valganciclovir oral $32 \mathrm{mg} / \mathrm{kg} / \mathrm{día}$ en dos dosis durante 6 meses (lo que facilita el tratamiento ambulatorio de estos niños), han demostrado disminuir las secuelas $(40,41)$. La tolerancia es buena y ha conseguido inhibir la replicación viral de forma prolongada, lo cual se ha relacionado con un mejor pronóstico neurológico (42-52) $(12,13)$. Con ambos tratamientos, el efecto adverso más frecuente es la neutropenia, que aparece en $2 / 3$ de los casos, por lo que se recomienda hemograma semanal mientras dure el tratamiento.
El seguimiento de estos niños con infección congénita debe ser multidisciplinar, llevar una vida normal y no deben tener medidas de aislamiento. Es fundamental iniciar tratamiento rehabilitador precoz cuando existe afectación del sistema nervioso central. Los controles se deben mantener anualmente hasta el final de la edad escolar aún estando asintomáticos.

\section{CONCLUSIÓN}

La presencia de hidrops fetal obliga a descartar entre sus posibles etiologías la infección congénita. EI CMV es la causa más frecuente de infección congénita en los países desarrollados y se relaciona con secuelas importantes. No se recomienda actualmente un cribado rutinario de CMV en el embarazo. Debe ser considerado para embarazadas con desarrollo de un síndrome pseudogripal o mononucleósico; mujeres con hijo en guarderías; trabajo en educación infantil; o sospecha ecográfica de lesiones compatibles. Tras el diagnóstico de infección congénita por CMV, el tratamiento precoz con ganciclovir intravenoso, ha demostrado disminuir las secuelas, especialmente la sordera neurosensorial y las alteraciones neurológicas, mejorando a largo plazo el pronóstico de estos niños. Valganciclovir oral, tiene efectos similares al ganciclovir, pudiendo ser una buena alternativa, facilitando el tratamiento ambulatorio. Sin embargo, son necesarios más estudios para definir la duración del tratamiento y sus efectos en la disminución de la sordera progresiva de estos pacientes.

\section{REFERENCIAS}

1. Kenneson A, Cannon MJ. Review and meta-analysis of the epidemiology of congenital cytomegalovirus (CMV) infection. Rev Med Virol 2007;17:253-76.

2. Ornoy A, Diav-Citrin O. Fetal effects of primary and secondary cytomegalovirus infection in pregnancy. Reprod Toxicol 2006;21:399-409.

3. Dollard SC, Grosse SD, Ross DS. New estimates of the prevalence of neurological and sensory sequelae and mortality associated with congenital cytomegalovirus infection. Rev Med Virol 2007;17:353-63.

4. Peckham C, Tookey P, Logan S, Guiaquinto C. Screening options for preventions of congenital cytomegalovirus infection. J Med Screen 2001;8:119-24.

5. Naessens A, Casteels A, Decatte L, Foulon W. A serologic strategy for detecting neonates at risck for congenital cytomegalovirus infection. J Pediatr 2005;146:194-7.

6. Barbi M, Binda S, Caroppo S, Calvario A, Germinario $\mathrm{C}$, Bozzi A, et al. Multicity Italian study of congenital cytomegalovirus infection. Pediatric Infect Dis J 2006;25:156-9. 
7. Malm G, Engman ML, Congenital cytomegalovirus infections. Semin Fetal Neonatal Med 2007;12:154-9.

8. Machin GA. Hydrops revisited: literature review of 1,414 cases published in the 1980s. Am J Med Genet 1989;34:366-90.

9. Instituto Nacional de Estadística. Movimiento natural de la población. Datos provisionales 2007. Disponible en: www. ine.es/prensa/NP507.pdf.

10. De Ory F, Castañeda R, Ramírez R, Pachón I. Estudio seroepidemiológico frente a citomegalovirus en mujeres en edad fértil de la Comunidad de Madrid. Med Clin (Barc) 1998;111:286-7.

11. De Ory F, Ramírez R, García-Comas L, León P, Sagües MJ, Sanz JC. Is there a change in cytomegalovirus seroepidemiology in Spain? Eur J Epidemiol 2004;19:85-9.

12. Protocolos Asistenciales de la Sociedad Española de Ginecología y Obstetricia Clínica (SEGO).Obstetricia. Protocolo n5, 2009. Disponible en: www.sego.es

13. De La Torre J, Sanfrutos L, González-González NL. Infecciones de trasmisión vertical (II). En: Bajo Arenas JM; Melchor Marcos JC; Mercé LT. Fundamentos de Obstetricia (SEGO). Madrid: Sociedad de Ginecología y Obstetricia y Laboratorios Meda-Pharma. 2007.

14. Pérez JL, Gimeno C, Navarro D, De Oña M, Pérez JL. Diagnóstico microbiológico de las infecciones por herpes virus. En: Cercenado E, Cantón R, editores. Procedimientos en microbiología clínica. Recomendaciones de la Sociedad Española de Enfermedades Infecciosas y Microbiología Clínica, 2005. [Citado 8 May 2009]. Disponible en: http://www.seimc.org/documentos/protocolos/microbiologia/indice8a.htm.

15. Yinon Y, Farine D, Yudin MH, Gagnon R, Hudon L, Basso $\mathrm{M}$, et al. Cytomegalovirus infection in pregnanacy. J Obstet Gynaecol Can 2010;32: 348-54.

16. Stagno S, Pass RF, Dworsky M.E. Congenital cytomegalovirus infection, the relative importance of primary and recurrent maternal infection. N Engl J Med. 1982; 306:945

17. Nigro G, Anceschi MM, Cosmi EV, and Congenital Cytomegalic Disease Collaborating Group. Clinical manifestations and abnormal laboratory findings in pregnant women with primary cytomegalovirus infection. BJOG 2003;110:572-7.

18. Pass RF, Fowler KB, Boppana SB, Britt WJ, Stagno $\mathrm{S}$. Congenital cytomegalovirus infection following first trimester maternal infection: symptoms at birth and outcome. J Clin Virol 2006;35:216-20.

19. Bodeus M, Hubinont $C$, Goubau P. Increased risk of cytomegalovirus transmission in utero during late gestation. Obstet Gynecol 1999;93:658-60.

20. Gindes L, Teperberg-Oikawa M, Sherman D, Pardo J, Rahav G. Congenital cytomegalovirus infection following primary maternal infection in the third trimester. BJOG 2008;115:830-5.

21. Pérez JL, Gimeno $C$, Navarro D, De Oña $M$, Pérez JL. Diagnóstico microbiológico de las infecciones por herpesvirus. En: Cercenado E, Cantón R, editores. Procedimientos en microbiología clínica. Recomendaciones de la Sociedad Española de Enfermedades Infecciosas y Microbiología Clínica, 2005. [Citado 8 May 2009]. Disponible en: http://www.seimc.org/docu- mentos/protocolos/microbiologia/indice8a.htm.

22. Lazzarotto T, Gabrielli L, Lanari M, Guerra B, Bellucci $\mathrm{T}$, Sassi $\mathrm{M}$, et al. Congenital cytomegalovirus infection: recent advances in the diagnosis of maternal infection. Hum Immunol 2004;65:410-5.

23. Lazzarotto T, Guerra B, Lanari M, Gabrielli L, Landini $\mathrm{N}$. New advances in the diagnosis of congenital cytomegalovirus infection. J Clin Virol 2008;41:192-7.

24. Bodeus M, Hubinont C, Bernard P, Bouckaert A, Thomas K, Goubau P. Prenatal diagnosis of human cytomegalovirus by culture and polymerase chain reaction: 98 pregnancies leading to congenital infection. Prenat Diagn 1999;19:314-7.

25. Adler SP, Nigro G, Pereira L. Recent advances in the prevention and treatment of congenital cytomegalovirus infections. Semin Perinatol 2007;31:10-8.

26. Guerra B, Simonazzi G, Puccetti C, Lanari M, Farina A, Lazzarotto T, Rizzo N. Ultrasound prediction of symptomatic congenital cytomegalovirus infection. Am J Obstet Gynecol 2008;198:380.e1-7.

27. Grose C, Weiner CP. Prenatal diagnosis of congenital cytomegalovirus infection: two decades later. Am J Obstet Gynecol 1990;163:447-50.

28. Lamy ME, Mulongo KN, Gadisseux JF, Lyon G, Gaudy V, Van Lierde M. Prenatal diagnosis of fetal cytomegalovirus infection. Am J Obstet Gynecol 1992;166:91-4.

29. La Torre R, Nigro G, Mazzocco M, Best AM, Adler SP. Placental enlargement in women with primary maternal cytomegalovirus infection is associated with fetal and neonatal disease. Clin Infect Dis 2006;15:994-1000.

30. Guerra B, Lazzarotto T, Quarta S, Lanari M, Bovicelli L, Nicolosi A, et al. Prenatal diagnosis of symptomatic congenital cytomegalovirus infection. Am J Obstet Gynecol 2000;183:476-82.

31. Fowler KB, McCollister FP, Dahle AJ, Boppana S, Britt WJ, Pass RF. Progressive and fluctuating sensorineural hearing loss in children with asymptomatic congenital cytomegalovirus infection. J Pediatr 1997;130:624-30.

32. Williamson WD, Demmler GJ, Percy AK, Catlin FI. Progressive hearing loss in infants with asymptomatic congenital cytomegalovirus infection. Pediatrics 1992;90:862-6.

33. Fowler KB, Dahle AJ, Boppana SB, Pass R.F. Newborn hearing screening: will children with hearing loss caused by congenital cytomegalovirus infection be missed? J Pediatr 1999;135:60-4.

34. Rivera LB, Boppana SB, Fowler KB, Britt WJ, Stagno $S$, Pass RF. Predictors of hearing loss in children with symptomatic congenital cytomegalovirus infection. Pediatrics 2002;110:762-7.

35. Boppana SB, Fowler KB, Pass RF, Rivera LB, Bradford RD, Lakeman FD, et al. Congenital cytomegalovirus infection: association between virus burden in infancy and hearing loss. J Pediatr 2005;146:817-23.

36. Lanari M, Lazzarotto T, Venturi V, Papa I, Gabrielli L, Guerra B, et al. Neonatal cytomegalovirus blood load and risk of sequelae in symptomatic and asymptomatic congenitally infected newborns. Pediatrics 2006;117:76-83.

37. Coats DK, Demmler GJ, Paysse EA, Du LT, Libby C. Ophthalmologic findings in children with congenital 
cytomegalovirus infection. J AAPOS 2000;4:110-6

38. Faqi AS, Klug A, Merker HJ, Chahoud I. Ganciclovir induces reproductive hazards in male rats after short term exposure. Hum Exp Toxic 1997;16:505-11.

39. Miller BW, Howard TK, Goss JA, Mostello DJ, Holcomb WL, Brennan DC. Renal transplantation one week after conception. Transplantation 1995;60:1353-4.

40. Pescovitz MD. Absence of teratogenicity of oral ganciclovir used during early pregnancy in a liver transplant recipient. Transplantation 1999;67:758-9.

41. Nigro G, Adler SP, La Torre R, Best AM, and Congenital Cytomegalovirus Collaborating Group. Passive immunization during pregnancy for congenital cytomegalovirus infection. N Engl J Med 2005;353:1350-62.

42. Kimberlin DW, Lin CY, Sánchez PJ, Demmler GJ, Dankner W, Shelton M, et al. Effect of ganciclovir therapy on hearing in symptomatic congenital cytomegalovirus disease involving the central nervous system: a randomized controlled trial. J Pediatr 2003;143:16-25.

43. Meine Jansen CF, Toet MC, Rademaker CM, Ververs TF, Gerards LJ, Van Loon AM. Treatment of symptomatic congenital cytomegalovirus infection with valganciclovir. J Perinat Med 2005;33:364-6.

44. Schulzke S, Buhrer C. Valganciclovir for treatment of congenital cytomegalovirus infection. Eur J Pediatr 2006;165:575-6.

45. Müller A, Eis-Hübinger AM, Brandhorst G, Heep A, Bartmann P, Franz AR. Oral valganciclovir for symptomatic congenital cytomegalovirus infection in an extre- mely low birth weight infant. J Perinatol 2008;28:74-6.

46. Baquero-Artigao F, Romero MP. Tratamiento prolongado con valganciclovir en un lactante con infección congénita por citomegalovirus. An Pediatr (Barc) 2009;70:578-81.

47. Galli L, Novelli A, Chiappini E, Gervaso MI, Csseta MI, Fallani S, et al. Valganciclovir for congenital CMV infection: a pilot study con plasma concentration in newborns and infants. Pediatric Infect Dis J 2007;26:451-3.

48. Kimberlin DW, Acosta EP, Sánchez PJ, Sood S, Agrawal V, Homans $\mathrm{J}$, et al. Pharmacokinetic and pharmacodynamic assesment of oral valganciclovir in the treatment of symptomatic congenital cytomegalovirus disease. J Infect Dis 2008;197:836-45.

49. Lombarda G, Grofoli F, Stronati M. Congenital cytomegalovirus infection: treatment, sequelae and followup. J Matern Fetal Neonatal Med 2010;23:45-8.

50. Rojo P, Ramos JT. Ganciclovir treatment of children with congenital cytomegalovirus infection. Pediatr Infect Dis J 2004;23:88-9.

51. National Institute of Allergy and Infectious Diseases (NIAID). Short-term vs. long-term valganciclovir therapy for symptomatic congenital CMV infections. [Citado 10 May 2009]. Disponible en: http://clinicaltrials.gov/ show/NCT00466817.

52. Amir J, Wolf DG, Levy I. Treatment of symptomatic congenital cytomegalovirus infection with intravenosus ganciclovir followed by long-term oral valganciclovir. Eur J Pediatr 2010;169:1061-7. 